\title{
Kinematics Rapid Modeling Method of Tendon-Driven Robotic Mechanisms Using the Tendon-Routing Matrix and Equivalent Radius Matrix
}

\author{
Hongqiang Sang ${ }^{1,2, *}$, Chenghao Yang ${ }^{1}$, Fen Liu ${ }^{1}$, Jintian Yun ${ }^{1}$ and Fa Chen ${ }^{1}$ \\ ${ }^{1}$ Key Lab for Advanced Mechatronics Equipment Technology Tianjin, China \\ ${ }^{2}$ School of Mechanical Engineering, Tianjin Polytechnic University, Tianjin, China \\ * Corresponding author
}

\begin{abstract}
A new kinematics rapid modeling method for $\mathrm{N}$-degree-of-freedom (DOF) serial tendon-driven robotic mechanisms with $\mathrm{N}, \mathrm{N}+1$, and $2 \mathrm{~N}$ configurations is proposed in this paper. Introducing the tendon-routing matrix (TRM) and equivalent radius matrix (ERM), which gotten by observing the routing topology of tendons, it is shown that all tendon routing can be described by two simple matrixes. The basic kinematic of tendon-driven robotic mechanism can be obtained by TRM and ERM. To avoid slackness in tendons and ensure to provide positive tendon tensions during the motion, the bias forces of the tendons is derived. To illustrate this method, the kinematic analysis of the Stanford /JPL finger is performed by TRM and ERM. The result shows that the proposed representation method can simplify kinematics modeling and analysis, significantly improve the kinematics modeling efficiency.
\end{abstract}

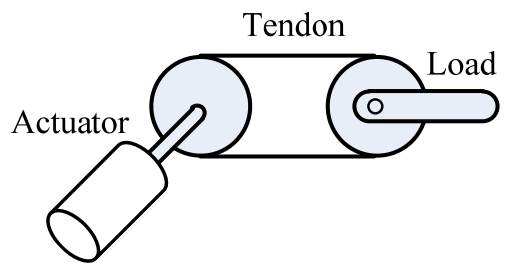

(a) $\mathrm{N}$ configuration

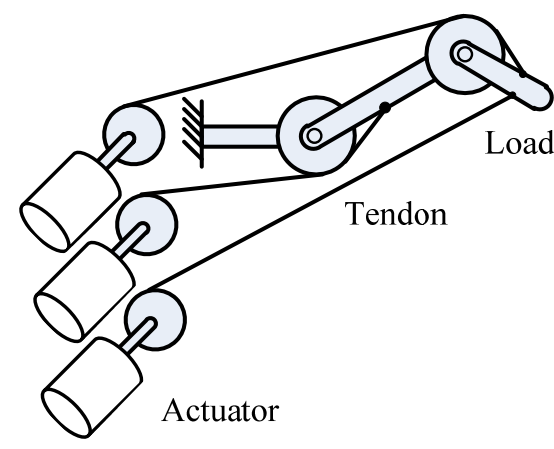

(b) $\mathrm{N}+1$ configuration

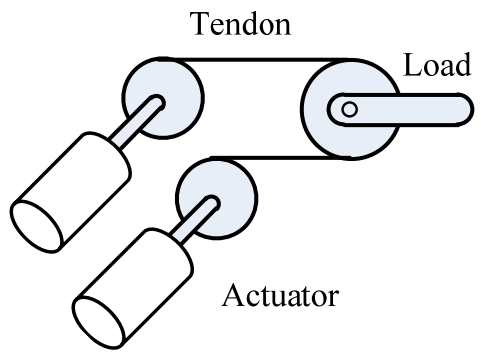

(c) $2 \mathrm{~N}$ configuration
FIGURE I. THE CLASSIFICATION OF SERIAL ACTIVE TENDON-DRIVEN ROBOTIC MECHANISMS
Serial active tendon-driven robotic mechanisms with $\mathrm{N}$ DOF can be classified into the $\mathrm{N}, \mathrm{N}+1$, and $2 \mathrm{~N}$ configurations. Fig 1(a) shows a single joint can be driven by one actuator with a pair of opposed tendons. A three fingered hand was developed applied this approach in actuation [7]. Fig 1(b) shows an example of the two DOF driven by three actuators with three tendons. Salisbury designed a three-fingered Stanford/JPL hand, and each finger has three DOF, which is actuated using four tendons [8].Fig 1(c) shows an example of the one DOF driven by two actuators with a pair of tendons. The Utah/MIT Dextrous Hand was developed by using the $2 \mathrm{~N}$ configuration [9].
Keywords-kinematics; tendon-driven; tendon-routing matrix; equivalent radius matrix

\section{INTRODUCTION}

Tendons have been widely used for motion and force transmission of robotic mechanisms in the past decades, such as tele-robots [1], parallel cable-suspended manipulators [2], dexterous hands [3], haptic[4], exoskeletons[5] and rehabilitation [6]. Compared with traditional transmission technology, tendon-driven configurations (cables, timing belts, chains, etc.) have advantages of large workspace, low backlash and friction, low weight and inertia, simplicity in structure, full remote actuation, reconfigurabilty and transportability.
To better design tendon-driven manipulators, a lot of researcher work was performed. The kinematics and statics of articulated tendon-driven robotics mechanisms were investigated [10]. The kinematics of tendon-driven robotic mechanisms was analyzed by using non-oriented graph technique. Uyguroğlu et al. investigated the kinematics structures of tendon-driven robotic mechanism with the aid of oriented graph. According to the oriented graph representation of kinematics structure, angular velocity, and static moment equations of tendon-driven robotic mechanisms can be systematically deduced [11]. The methodology for the kinematic synthesis of tendon-driven manipulators with isotropic transmission characteristics was developed [12]. 
For the kinematics analysis based on graph theory, it is necessary to transform three-dimensional kinematics structure of the tendon-driven robotic mechanisms into two-dimensional graph representation. For the kinematics analysis based on non-oriented graph technique, fundamental circuit equations must be constructed for each pulley based on transmission lines. Combined with coaxiality conditions, the kinematic mapping relationship of tendon displacements and joint angular displacements can be obtained.

Combined with [13], the kinematics analysis of tendon-driven robotic mechanisms can be divided into two fundamental parts: The first part is kinematics analysis of open-loop robot removal of the tendons and pulleys, to denote the mapping relationship between joint space and Cartesian space. The second one is the mapping relationship between joint space and actuator space with tendons and pulleys, which will be analyzed in this paper.

\section{THE BASIC KINEMATICS OF TENDON-DRIVEN RoBOtIC MECHANISMS}

\section{A. General Assumptions}

To adapt the scope of this work, tendon-driven robotic mechanisms will abide by the following assumptions [14]:

(1) All tendons are under tension and the amount of stretch in tendons due to tension is ignored.

(2) The frictions between tendons and pulleys are large enough to prevent relative slippage happening.

(3) The mechanism is of articulated type. It becomes an open-loop chain after the removal of tendons and pulleys, and obeys the general DOF equations.

(4) Each pulley has a revolving pair on its axis, and to keep a constant center distance, every pair of pulleys is connected by a carrier.

\section{B. Kinematics of Transmission}

Figure 2 shows an active tendon driven by an actuator.

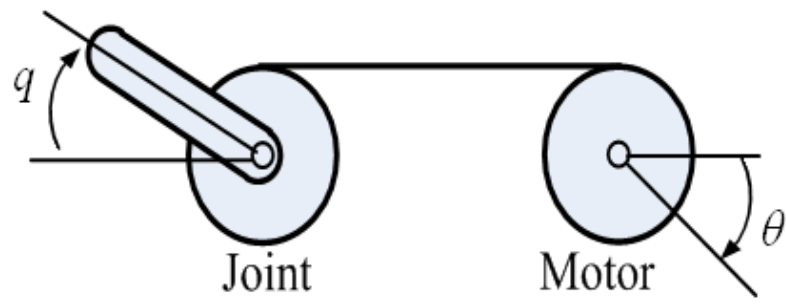

FIGURE II. ACTIVE TENDON DRIVEN BY AN ACTUATOR

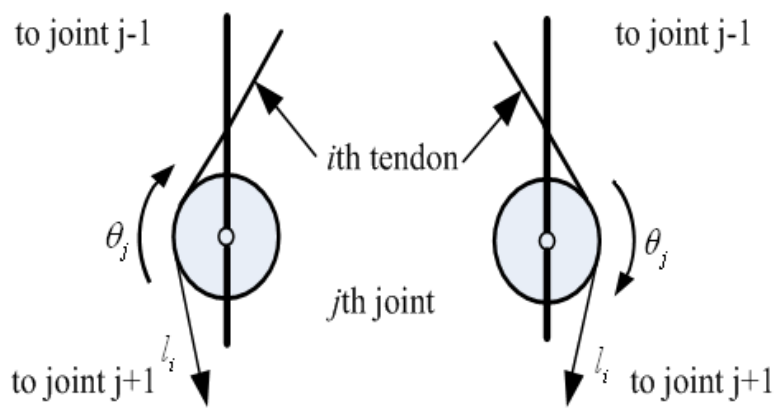

$\begin{array}{ll}\text { (a) Negative } r_{i j} & \text { (b) Positive } r_{i j}\end{array}$

FIGURE III. THE SIGN DEFINE OF $r_{i j}$

Let

$\boldsymbol{l}=\left(l_{1}, l_{2}, \cdots, l_{k}\right) \in R^{k}, \boldsymbol{\theta}=\left(\theta_{1}, \theta_{2}, \cdots, \theta_{m}\right) \in R^{m}, \boldsymbol{q}=\left(q_{1}, q_{2}, \cdots, q_{n}\right) \in R^{n}$ be the tendon displacement, the actuator rotation angular displacement, and joint angular displacement vectors respectively, where $\mathrm{k}, \mathrm{m}$, and $\mathrm{n}$ are the numbers of tendons, actuators, and joints of the tendon-driven robotic mechanism respectively. The relationship among $\boldsymbol{l}, \boldsymbol{\theta}$ and $\boldsymbol{q}$ can be described by

$$
l=l(q)=l(\theta)
$$

The time derivative of the displacement vector can be expressed as

$$
\boldsymbol{l}=\boldsymbol{J}_{t} \boldsymbol{q}=\boldsymbol{R}_{a} \boldsymbol{\theta}
$$

where $\boldsymbol{J}_{t}$ is a $k \times n$ dimensional tendon Jacobian, $\boldsymbol{R}_{a}$ is a $k \times m$ dimensional matrix, whose diagonal elements are the radii of pulleys attached on the actuators.

According to the virtual work principle, the transformation between the tendon tension forces and the joint torques can be written as

$$
\boldsymbol{\tau}=\boldsymbol{J}_{t}^{\mathrm{T}} \boldsymbol{f}_{t}
$$

The tendon tensile force can be expressed as

$$
\boldsymbol{f}_{t}=\left(\boldsymbol{J}_{t}^{\mathrm{T}}\right)^{+} \boldsymbol{\tau}+\boldsymbol{H} \boldsymbol{\lambda}
$$

where $\left(\boldsymbol{J}_{t}^{\mathrm{T}}\right)^{+}=\boldsymbol{J}_{t}\left[\boldsymbol{J}_{t}^{\mathrm{T}} \boldsymbol{J}_{t}\right]^{-1}$ is the pseudo-inverse of $\boldsymbol{J}_{t}^{\mathrm{T}}$, $\boldsymbol{H}$ is a $k \times(k-n)$ dimensional matrix and the column vectors can span the null space of matrix $\boldsymbol{J}_{t}^{\mathrm{T}}, \boldsymbol{\lambda}$ with arbitrary 
constant elements is a $(k-n) \times 1 \quad$ vector. When $\operatorname{rank}\left(\boldsymbol{J}_{t}^{\mathrm{T}}\right)=n$, the first item on the right-hand side of Eq. (4) is the particular solution and the second term is the homogeneous solution. The homogeneous solution $\boldsymbol{f}_{b}=\boldsymbol{H} \boldsymbol{\lambda}$ is used to adjust the tension in tendons, called as bias force, which has no effect on the resultant joint torques and allows all tendons to remain in position tension.

\section{TENDON-ROUTING MATRIX AND EQUIVALENT RADIUS MATRIX}

\section{A. Functional Representation}

In order to introduce tendon-routing matrix and equivalent matrix, functional representation is defined. The essential functional elements of the conventional drawing of a mechanism such as tendons, joint shafts, actuator units, driving pulleys installed on the output axes of actuator unit and driven pulleys connected with tendons and installed on the joint axes are usually considered in the drawing. And define the positive direction of tendon displacement to point to the motor driven unit, and choose the positive direction of joint and motor rotor angular displacements.

\section{B. Tendon-routing Matrix}

To describe the tendon routing for tendon-driven robotic mechanism, a TRM is introduced. $\boldsymbol{J}_{t}$ is the tendon Jacobian and also called as TRM, whose elements are functions of tendon routings and radius of pulleys mounted on the resultant joints.

$$
\boldsymbol{J}_{t}=\left[r_{i j}\right] \text { is a } k \times n \text { dimensional matrix, where the rows }
$$
are correspond to tendons $l_{1}, l_{2} \cdots l_{k}$, and the columns are correspond to joint axes $\bar{Z}_{1}, \bar{Z}_{2} \cdots \bar{Z}_{n}$. The element $r_{i j}$ describes the condition of adjacency of the joint $j$ and tendon $i$. If the $i$ th tendon is not routed about the $j$ th joint, the valve of the elements $r_{i j}$ is zero. If the $i$ th tendon is routed about the $j$ th joint, the absolute valve of the element $r_{i j}$ is the radius of the pulley attached on the $j$ th joint and routed by the $i$ th tendon. If tendon $i$ produces a positive displacement, and the pulley attached on joint $j$ driven by tendon $i$ rotates in the same positive direction with the joint angular displacement, the sign of the element $r_{i j}$ is positive, otherwise it is negative. Fig 3 shows the sign definition of $r_{i j}$.

\section{Equivalent Radius Matrix}

$\boldsymbol{R}_{a}$ is a $k \times m$ dimensional matrix, whose elements are the radii of pulleys attached on the actuators. When a reducer with reduction ratio $\mathrm{n}$ is attached on an actuator, the elements of matrix are specific value of the radii of pulleys attached on the reducer and reduction ratio. Thus, $\boldsymbol{R}_{a}$ is also called as equivalent radius matrix. For the sake of simplification, let reduction ration $\mathrm{n}$ be equal to one in this paper. For $\mathrm{N}+1$ and $2 \mathrm{~N}$
Configurations, ERM is a diagonal matrix, whose diagonal elements are the radii of pulleys attached on the actuators. For $\mathrm{N}$ Configuration, ERM is a generalized diagonal matrix,

$$
\boldsymbol{R}_{a}=\operatorname{diag}\left\{\begin{array}{llll}
\boldsymbol{D}_{1} & \boldsymbol{D}_{2} & \cdots & \boldsymbol{D}_{n}
\end{array}\right\}
$$

and $\boldsymbol{D}_{i}=\left[\begin{array}{ll} \pm r_{a i} & \mp r_{a i}\end{array}\right]^{\mathrm{T}} i=1,2, \cdots, n, r_{a i}$ is the radius of pulley attached on the $i$ th actuator. If the tendon reference direction positive direction of the actuator unit and is the same, the sign of the element is positive, otherwise it is negative.

\section{EXAMPLES}

The method is universally applicable for $\mathrm{N}, \mathrm{N}+1$ and $2 \mathrm{~N}$ Configurations. It is illstrated in this paper with one example of the Stanford/JPL finger which is a typical tendon-driven mechanism with $\mathrm{N}+1$ configuration. The functional representation of the finger is shown in Figure 4.

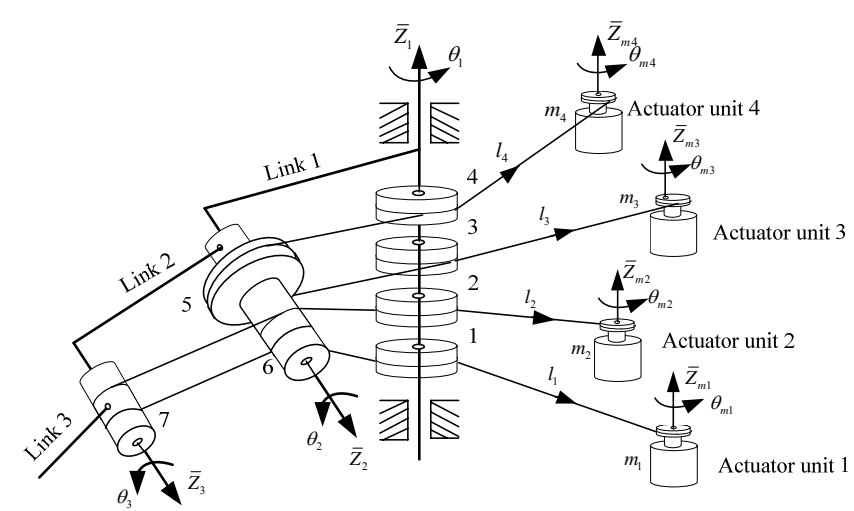

FIGURE IV. FUNCTIONAL REPRESENTATION OF THE STANFORD/JPL FINGER

The first joint axis $\bar{Z}_{1}$ is fixed to the base, the second joint axis $\bar{Z}_{2}$ is perpendicular to the first, and the third joint axis $\bar{Z}_{3}$ is parallel to the second. Driven pulleys $1,2,3,4$, driven pulleys 5,6 , and driven pulley 7 are free to rotate about the axis $\bar{Z}_{1}, \bar{Z}_{2}$ and $\bar{Z}_{3}$, respectively. Link 1 , link 2 and link 3 serve as the carriers for driven pulley pairs $(4,5),(3,5)(2,6)$, $(1,6)$, driven pulley pair $(6,7)$, and driven pulley7, respectively. Driving pulleys $m_{1}, m_{2}, m_{3}$ and $m_{4}$ are fixed to output axis $\bar{Z}_{m 1}, \bar{Z}_{m 2}, \bar{Z}_{m 3}$ and $\bar{Z}_{m 4}$ of actuator units $1,2,3,4$, respectively. Assume the positive direction of tendon displacement point to actuator unit, and the positive directions of joint angular displacements and angular displacements of actuator units are anticlockwise.

According to functional representation of the Stanford/JPL finger, TRM and ERM can be written as 


$$
\boldsymbol{J}_{t}=\left[\begin{array}{ccc}
-r_{1} & r_{6} & r_{7} \\
-r_{2} & -r_{6} & -r_{7} \\
r_{3} & r_{5} & 0 \\
r_{4} & -r_{5} & 0
\end{array}\right] \quad \boldsymbol{R}_{a}=\left[\begin{array}{cccc}
-r_{m 1} & 0 & 0 & 0 \\
0 & -r_{m 2} & 0 & 0 \\
0 & 0 & r_{m 3} & 0 \\
0 & 0 & 0 & r_{m 4}
\end{array}\right]
$$

The basic kinematics of the Stanford/JPL finger can be described as

$$
\boldsymbol{J}_{t} \boldsymbol{q}=\boldsymbol{R}_{a} \boldsymbol{\theta}
$$

where

$$
\boldsymbol{q}=\left[\begin{array}{lll}
\theta_{1} & \theta_{2} & \theta_{3}
\end{array}\right]^{\mathrm{T}}, \boldsymbol{\theta}=\left[\begin{array}{llll}
\theta_{m 1} & \theta_{m 2} & \theta_{m 3} & \theta_{m 4}
\end{array}\right]^{\mathrm{T}}
$$

For simplicity, the radii of all the pulleys in TEM and ERM are assumed to be unified. The bias forces of the tendons can be expressed as $\boldsymbol{f}_{b}=\left[\begin{array}{llll}1 & 1 & 1 & 1\end{array}\right]^{\mathrm{T}}$.

\section{CONCLUSIONS}

A kinematics rapid modeling method for N-DOF serial tendon-driven robotic mechanisms with $\mathrm{N}, \mathrm{N}+1$, and $2 \mathrm{~N}$ configurations is put forward. The TRM and ERM can be obtained by observing the routing topology of tendons in the functional representation. The basic kinematic of tendon-driven robotic mechanism can be obtained by TRM and ERM and the bias forces of the tendons can also be obtained. To illustrate this method, the kinematic of the tendon-driven robotic mechanisms with $\mathrm{N}+1$ configuration is analyzed by TRM and ERM. The result shows that the proposed method can simplify kinematics modeling and analysis, significantly improve the kinematics modeling efficiency.

\section{ACKNOWLEDGMENTS}

The work is supported by National Natural Science Foundation of China (No.51205287).

\section{REFERENCE}

[1] A.J. Madhani, G. Niemeyer, J.K. Salisbury. The Black Falcon: a teleoperated surgical instrument for minimally invasive surgery, in: IEEE/RSJ International Conference on Intelligent Robots and Systems, 1998, 936-944.

[2] S. Kawamura, H. Kino, C. Won. High-speed manipulation by using parallel wire-driven robots, Robotica , 2000,18, 3-21.

[3] S. Narasimhan. Dexterous robotic hands: kinematics and control, Massachusetts Institute of Technology, Cambridge Artificial Intelligence Lab, 1988.

[4] F. Ferlay and F. Gosselin. A new cable-actuated haptic interface design, in Proc. Int. Conf. Hapt.: Percept., Devices, Scenarios, 2008, 474-483.

[5] S. K. Agrawal, V. N. Dubey, J. J. Gangloff, et al. Design and optimization of a cable driven upper arm exoskeleton, J. Med. Dev., 2009, 3, 031004-1-031004-8,

[6] Y. Mao and S. K. Agrawal. Design of a cable-driven arm exoskeleton (CAREX) for neural rehabilitation, IEEE Trans. Robot., 2012, 28(4), 922-931.

[7] T. Okada. On a Versatile Finger System, Proc. 7th ISIR, 1977, 345-352.

[8] J. K. Salisbury, J. J. Craig. Articulated Hands: Force Control and Kinematic Issues, International Journal of Robotics Research, 1982,1(1), 4-17.
[9] S. C. Jacobsen, J. E. Wood, D. F. Knutti, et al. The Utah/MIT Dextrous Hand Work in Progress, The International Journal of Robotics Research, 1984,4(3), 21-50.

[10] J. K. Salisbury. Kinematic and Force Analysis of Articulated Hands, Stanford, CA: Department of Mechanical Engineering, Stanford University, 1982.

[11] M. Uyguroğlu, H. Demirel, Gazimagusa, et al, Kinematics analysis of tendon-driven robotic mechanisms using oriented graphs, Acta Mechanica, 2006,182, 265-277.

[12] Y. J. Ou, L. W. Tsai. Kinematic synthesis of tendon-driven manipulators with isotropic transmission characteristics, ASME J. Mech. Des.,1993,115, 884-891.

[13] L. W. Tsai. Robot Analysis: The Mechanics of Serial and Parallel Manipulators, John Wiley \& Sons, Inc., New York, 1999.

[14] H. Q. Sang , S. X. Wang, J. M. Li, et al. Control Design and Implementation of a Novel Master-Slave Surgery robot system MicroHand A, Int J Med Robotics Comput Assist Surg, 2011, 7( 3), $334-347$ 